\title{
The European Synchrotron Radiation Facility in Grenoble
}

\author{
M. Altarelli \\ European Synchrotron Radiation Facility, Grenoble, France \\ An Associate Member of the EPS
}

\begin{abstract}
Electron or positron storage rings are the most powerful sources of ultraviolet and $\mathrm{X}$-ray photons available to experimentalists interested in spectroscopic and structural studies in condensed matter as well as in molecular and atomic systems. The use of synchrotron radiation over the last twenty years has revolutionized $\mathrm{X}$-ray physics and its application to materials science, surface science, crystallography, chemistry, structural biology and other fields of both fundamental and applied significance.
\end{abstract}

The impressive record of scientific achievements made possible by synchrotron radiation has stimulated a considerable effort in the design and optimization of dedicated sources. The most recent generation of sources, which are all under construction or on the drawing board, are characterized by two features: one is the very low emittance, generally $\leq 10 \mathrm{~nm}$.rad in the horizontal plane (as we shall see below, this quantity expresses the size and the angular divergence of the particle beam circulating in the storage ring); the other is the extensive use of insertion devices (wigglers and undulators) as radiation sources. The combined effect of the low-emittance, corresponding to a reduced source size and collimation, and of insertion devices, with improved radiation properties, results in an increase of many orders of magnitude in brilliance of the new sources with respect to existing ones.

The machines of this new generation can be divided in two groups, differing in the energy of the circulating electrons or positrons. One group of sources is planned to have an energy 1-2 GeV, optimized for the VUV and soft X-ray spectral ranges; the Advanced Light Source in Berkeley (USA), Elettra in Trieste (Italy) and Bessy II in Berlin belong to this category. Another type of source, optimized for the hard X-ray

Dr. Massimo Altarelli is the Research Director of the European Synchrotron Radiation Facility, BP 220, F-38043 Grenoble, France. part of the spectrum, is represented by the planned Advanced Photon Source (APS) in Argonne (USA) with an energy of $7 \mathrm{GeV}$, by the Japanese $8 \mathrm{GeV}$ project in the Kansai region, and by the European Synchrotron Radiation Facility (ESRF) with $6 \mathrm{GeV}$ which is under construction in Grenoble.

A machine already in operation should be added to the list: it is the PEP ration was demonstrated at an energy of $7 \mathrm{GeV}$, with a horizontal emittance of the order of $10 \mathrm{~nm} . \mathrm{rad}$, and on which undulators are installed for parasitic and dedicated exploitation.

The purpose of this article is to provide a short description of the Grenoble ESRF project. The main features of the ESRF are discussed, together with a few examples of the new scientific possiblities that it will open up. It is important to mention that the ESRF project is supported, at the time of writing, by eleven European countries: France, the Federal Republic of Germany, Italy, UK, Spain, Switzerland, Belgium, Denmark, Finland, Norway and Sweden. Other countries may associate themselves to the project in the near future: the Netherlands, in particular, has initiated a formal procedure to this effect.

\section{Source Parameters}

We summarize here the main features of the ESRF synchrotron radiation source (the interested reader can find a thorough description of the design, finalized in February 1987, in the ESRF Foundation Phase Report [1]). It is useful to recall $[1,2]$ that the spectrum emitted by the bending magnets which hold electrons or positrons on a circular orbit is continuous, with a high-energy storage ring in Stanford, for which ope-

cutoff above the critical $E_{\mathrm{c}}$ where

$$
E_{\mathrm{c}}(\mathrm{keV})=0.6651 \mathrm{BE}^{2}
$$

Here $B$ is the magnetic field (in tesla) and $E$ the energy (in $\mathrm{GeV}$ ) of the machine. The total radiated power $P$ (in $\mathrm{kW}$ ) is:

$$
P(\mathrm{~kW})=0.2654 B E^{3} I
$$

where $I$ is the circulating current (in amperes). The radiation is emitted in a narrow cone in the tangential direction and the angular aperture in the vertical plane equals the inverse of the relativistic parameter $\gamma=\left(1-v^{2} / c^{2}\right)^{-1 / 2}$.

\section{Insertion Devices}

In the new generation synchrotron sources, the emission of radiation takes place not only from the bending magnets, but mostly from the so-called insertion devices (undulators or wigglers) installed in straight sections of the storage ring. An insertion device consists generally of an array of magnets producing steady vertical magnetic fields, with a sinusoidal position dependence in the direction of the electron motion (see Fig. 1). The oscillatory field forces the electrons to follow a zig-zag trajectory, with many sharp curves, from which high intensity radiation is emitted. There are two basic advantages with respect to bending magnets: first, any magnetic field can be adopted in the device, independent of that adopted for the bending magnets of the ring; second, since many "wiggles" can be produced along the trajectory, the radiated flux is increased accordingly with respect to that for a series of bending magnets.

Undulators are insertion devices with a small field, producing small angular deviations (compared with the characteristic emission angle $1 / \gamma$ ) of the tra-

Fig. 1 - Schematic view of the magnetic structure of an insertion device. Also shown are the electron trajectory, the maximum angle of deviation $\alpha$, and one radiation cone (of aperture $1 / \gamma$ ) from a particular point on the trajectory.

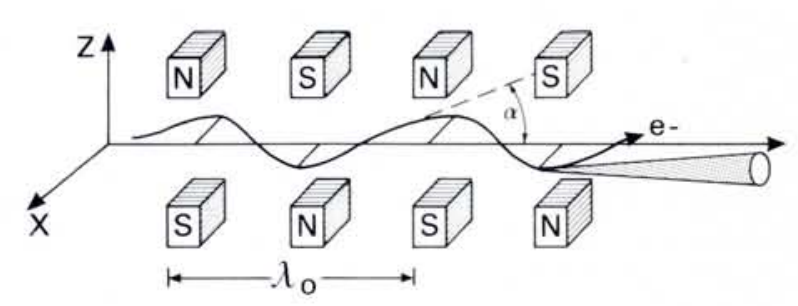


jectory from a straight line. The emission cones from successive bends of the trajectory overlap, producing, by interference phenomena, a spectrum of lines with $\Delta E / E \sim 1 / N$, where $N$ is the number of magnetic periods in the device. High field devices, called wigglers, produce angular deviations much greater than $1 / \gamma$ resulting in a broad emission band. For one wiggler with $N$ periods each of length $\lambda_{0}$ (in $\mathrm{cm}$ ), and a peak field $B_{m}$, the total radiated power is

$$
P(\mathrm{~kW})=0.00633 B_{\mathrm{m}}^{2} E^{2} N \lambda_{0} l
$$

where all units are as in Eq. (1).

The energy of the ESRF was chosen as $6 \mathrm{GeV}$ to meet the specifications for high flux and high critical energy (19.2 $\mathrm{keV}$ from the $0.8 \mathrm{~T}$ bending magnets and up to $14.4 \mathrm{keV}$ in the first harmonic of undulators), and for a wide tunability range of the undulators (see below).

\section{Emittance}

It may also be useful to remember that the horizontal emittance is defined $[2,3]$ as the area in phase space $\left(x, x^{\prime}\right)$ which encompasses a specified fraction (one standard deviation) of the number of circulating particles. Here $x$ denotes the distance in the radial direction between the position of a real orbit and the ideal orbit, while $x^{\prime}$ is the angle between the velocity of the particle on the real orbit with respect of the ideal one (the $x, x^{\prime}$ values of all electrons at one particular section are expected to have a Gaussian distribution around the ideal values). This area is a constant of the motion, i.e. it does not depend on the particular point of the trajectory where it is evaluated. Similarly, the vertical emittance is defined in terms of the $\left(z, z^{\prime}\right)$ phase space area, where $z$ is the direction normal to the orbit plane. The emittance is thus a measure of the size and angular spread of the particle beam, and is related to the brilliance, defined as the number of photons emitted by the source per second, per unit source area, per unit solid angle, and per $0.1 \%$ bandwidth around a given frequency. High brilliance is important for many experiments (see below), and is the reason for the requirement of low emittance, i.e. small source size. The horizontal emittance of the ESRF has been specified to be $7 \mathrm{~nm}$.rad, and the vertical emittance is one order of magnitude smaller. This performance is one to two orders of magnitude better than for existing dedicated sources.

\section{Design Parameters}

The horizontal emittance of a storage ring is a property of the magnetic lattice. The lattice adopted for the ESRF is the result of an optimization process sub- ject, besides the emittance and energy requirements, to the additional constraints of a circumference not exceeding $850 \mathrm{~m}$, of a large number of straight sections for the installation of insertion devices, and of low sensitivity to errors. It is a lattice of the Chasman-Green type [4], with 32 straight sections of $6 \mathrm{~m}$ free length, 29 of which can accommodate insertion devices (two are needed for the RF cavities and one for injection from the booster synchrotron).

The current in the storage ring is specified to be in excess of $0.1 \mathrm{~A}$, without reducing the lifetime below $10 \mathrm{~h}$. It is not possible to say at present if the latter requirement will impose the use of positrons, instead of electrons. Positrons repel positive ions created in the residual gas, thus avoiding ion trapping which is detrimental to the stability of electron beams. The quoted value for the current is for the multi-bunch mode of operation, in which the particles circulate around the ring in 992 bunches or packets. Single-bunch operation is possible, but with a current reduced by one order of magnitude.

The total circumference of the storage ring is $844 \mathrm{~m}$. Inside the main ring there will be a $200 \mathrm{MeV}$ linear accelerator for electrons, and possibly an $\mathrm{e}^{-} / \mathrm{e}^{+}$ converter and a $400 \mathrm{MeV}$ positron linac, which will feed the particles into a booster synchrotron where their energy is raised to $6 \mathrm{GeV}$, for injection into the storage ring.

The brilliance of ESRF bending magnets and typical wiggler and undulator sources is shown in Fig. 2 as a function of the photon energy. For users interested in experiments requiring soft X-rays, the high critical energy of the $0.8 \mathrm{~T}$ bending magnets may not be desirable. For this reason, a short dipole (length 20 $\mathrm{cm}$, field $0.4 \mathrm{~T}$ ) is placed at the entrance of the main dipole. The field vs. angle profile then has a plateau of $0.4 \mathrm{~T}$ over 4 mrad. The beam line can be thus aligned so as to extract radiation from either the $0.8 \mathrm{~T}$ or the $0.4 \mathrm{~T}$ regions.
For undulators of a given period $\lambda_{0}$, the energy of the fundamental and higher harmonics depends on the field, which can be tuned by varying the gap between the upper and lower sets of magnetic poles (see Fig. 1). Tunability is one of the most important factors to be considered in the process of defining the optimum undulator sources for a given instrument. The curves in Fig. 2 show the tunability ranges of the first and third harmonics of various undulators. A remarkable increase in brilliance for insertion devices with respect to bending magnets is evident.

\section{Scientific Programmes}

It is foreseen that commissioning of instruments at ESRF will start in mid1993. A first group of seven beam lines will be operating for external users in mid-1994; within the following 12 months it is planned that 11 more beam lines will be commissioned. Three new beam lines will then be brought into operation each year, up to a total of 30 by 1999 . Although the construction of such a large number of instruments is a major technical challenge, requiring the mobilization and collaboration of all Europe's expertise, the bulk of the instrumentation of the beam lines will be built within the budget of the facility. The lines will be operated and made available to users free of charge, on the basis of scientific merit. It is furthermore important to note that since there are 29 straight sections for insertion devices, plus 27 bending magnet ports, the 30 "public" beam lines are far from exhausting the potential of the machine, especially in so far as bending magnet sources are concerned (most of the 30 "public" beam lines will be on the insertion devices). The ESRF is receiving and considering proposals from groups of users intending to build additional instruments at ESRF with independent funding; they will, in exchange, receive privileged access to a large fraction of the available beam time.
Fig. 2 - Brilliance of some ESRF sources as a function of the photon energy.

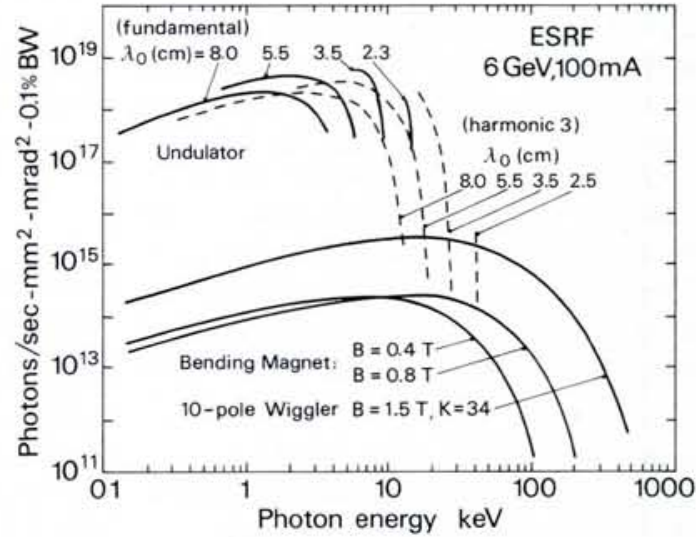


The scientific interest of ESRF encompasses many fields of science ranging from crystallography to surface science, from materials research to biology and medicine, from nuclear physics to earth sciences, etc. The choice of beam lines and their priority schedule is therefore a complex task, for which input from potential users from the entire European scientific community plays a crucial role. Over 200 such users convened in Grenoble on 20-22 March 1989 to discuss scientific priorities. Many interesting scientific cases were presented, in fact too many to be accommodated at the very beginning. With further help from the 22 members of the ESRF Scientific Advisory Committee, a priority list for the first 18 "public" beam lines was established in July 1989. The scientific goals for the first eight beam lines, for most of which the design phase is well under way, will be briefly described.

\section{Microfocus Beam Line}

This instrument will use the exceptional properties of high brilliance undulator radiation to produce a focal spot size of the order $10 \mu \mathrm{m} \times 10 \mu \mathrm{m}$. Further collimation of the beam by slits could allow reduction to $1 \mu \times 1 \mu$. Scientific uses include microcrystal diffraction, small angle scattering and microtomography, with photon energies up to $\cong 25 \mathrm{keV}$.

2. Materials Science Wiggler Beam Line Using the intense wiggler radiation up to $40 \mathrm{keV}$, this beam line will provide tunable monochromatic radiation by a fixedexit, double crystal monochromator to allow high-momentum resolution crystallographic studies in materials science, and other applications of diffraction. The experimental station will be equipped with a high stability 6-circle diffractometer.

\section{Laue Protein Crystallography}

A wiggler source similar to that of the previous beam line will be used to obtain white beam Laue diffraction patterns of macromolecular samples. This technique is one of the most promising for structural studies of biological molecules. The ESRF source will reduce exposure time, thus limiting radiation damage, and improve angular resolution. If supported by advances in position-sensitive detector technology, this instrument could make a very significant contribution not only to the solution of these very complex structures, but also to kinetic studies of the dynamics of biological reactions. In addition, the white beam will be used for energy dispersive diffraction studies of materials under high hydrostatic pressure.

\section{High-Brilliance Undulator Beam Line}

The source for this beam line is optimized to produce the highest brilliance, at the expense of tunability. It will therefore operate in a narrow wavelength range, in the neighborhood of $1 \AA$, and will be used for real-time small angle scattering applications (with possible applications to muscle dynamics, interface dynamics, etc.) and for monochromatic protein crystallography.

\section{High-Energy X-Ray Scattering Beam Line}

A high field wiggler on ESRF can generate a critical energy in excess of $80 \mathrm{keV}$. At such energies, the absorption corrections become very small and X-ray scattering can probe large volumes of the sample (as in neutron scattering). Studies of crystalline perfection and of the distribution of elastic strains in large crystals become possible. Compton scattering using such an intense source will become more important in the study of the electronic properties of solids.

\section{Circular Polarisation Beam Line}

Specially designed undulators may ge nerate a magnetic field with vertical and horizontal components, forcing electrons to follow a helical trajectory and resulting in emission of radiation with more than $90 \%$ of circular or elliptic polarization for energies up to a few $\mathrm{keV}$ in the first harmonic [5]. Absorption and photoemission studies with circularly polarized photons can provide very significant and novel in formation on magnetic materials, on surface magnetism, and on chiral molecules of importance in chemistry and biology.

\section{Surface and Interface Diffraction Beam} Line

Grazing incidence diffraction has proved to be a very powerful tool for the in vestigation of long-range order, phase transitions, growth mechanisms and reconstruction phenomena in surface physics. The high brilliance up to $25 \mathrm{keV}$ photon energy from an ESRF undulator will improve resolution, offer increased sensitivity to low-coverage and light-atom adsorbates, and allow access to buried interfaces in layered structures, in systems of electrochemical interest, etc.

\section{Dispersive EXAFS}

EXAFS is a technique for obtaining structural information on the short-range neighborhood of a given atom by measuring the absorption coefficient in an interval of a few hundreds of eV's above one of the atomic core absorption thresholds. In the dispersive mode, the whole spectrum in this region is collected simultaneously using dispersive optics with a positionsensitive linear detector. Detector readout-time limits to $\cong 1 \mathrm{~ms}$ the acquisition time; this is, however, sufficient in some cases to follow slow processes that modify the atomic environment, e.g. catalyst reactions.

The second generation of ESRF beam lines will also cater for fields of high interest such as inelastic scattering, Mössbauer diffraction, magnetic scattering, $\mathrm{X}$-ray topography and many others. This brief and incomplete survey hopefully conveys the breadth of the new and exciting fundamental research which will be possible at the ESRF. Fur- thermore, structural studies of systems relevant to the microelectronic, chemical and pharmaceutical industries also fall very much within the ESRF's overall objectives.

\section{Project Status}

The international convention that gave official birth to the ESRF was signed in December 1988. But well before that date a nucleus of the project staff had settled in Grenoble, on a site adjacent to the high-flux reactor of the Institute Laue-Langevin (ILL). The ESRF staff today numbers 130 and will gradually grow to 402 by 1995 , at which time the ESRF will also support $32 \mathrm{PhD}$ students performing their thesis work.

The ring site was excavated and levelled during the early part of 1989. A large contract for the construction of the linear accelerator building, the booster synchrotron building, the storage ring and experimental hall building and some technical utilities buildings was awarded to a consortium of European firms and was signed in December 1989. Construction work began a few weeks later and will be completed by the end of 1991. The central building with staff offices, some laboratories and meeting and lecture halls, and an additional building to be shared with ILL comprising the cafeteria, the central library and offices for the theory groups will be put to tender during 1990. As of today about $50 \%$ of the capital budget for the machine has been committed. Key components such as the complete linear accelerator, and the magnets and the radio-frequency systems for the synchrotron and the storage ring have been ordered. The total investment for the construction of ESRF amounts to 1.7 billions of French Francs.

The first beam is expected to be stored at the end of 1992. As the first photons emerge from the insertion devices in 1993, European scientists will have an exceptional instrument at their disposal, delivering its scientific pay-off for many years to come.

\section{REFERENCES}

[1] ESRF Foundation Phase Report (Grenoble) 1987.

[2] Winick H. in: Synchrotron Radiation Research, Eds H. Winick and S. Doniach (Plenum, New York, 1980) 11.

[3] Koch E.E., Eastman D.E. and Farge Y. in: Handbook of Synchrotron Radiation, Vol. 1a, Ed. E.E. Koch (North Holland, Amsterdam, 1983) 1.

[4] See Ref. 1, especially Chapter II, for details of the lattice structure.

[5] Elleaume P., Report ESRF/ID 88-23, to appear in: "Proceedings of the IX Int. Conf. on VUV Radiation Physics, Hawaii, 17-21 July 1989"; Physica Scripta (1990). 